\title{
Evaluation of Cardiac Coronary Artery in Children with Kawasaki Disease by Echocardiography
}

\section{zич Lin}

Capital Pediatric Institute Affiliated Children 's Hospital, Beijing 100000

Abstract: Kawasaki disease is an acute, unknown etiology of vascular inflammatory response syndrome, heart coronary artery changes as one of the main clinical manifestations of Kawasaki disease. How to determine the effective changes of coronary heart disease in children has become a lot of research scholars concern. With the continuous development of medical technology in recent years, echocardiography is widely used in the clinical diagnosis of various diseases and for the effective development of clinical treatment had a positive impact. A large number of research scholars found that by echocardiography, it can effectively achieve the diagnosis of coronary heart disease in children with Kawasaki disease, in order to lay a good foundation for the development of clinical treatment.

Key words: echocardiography; Kawasaki disease; heart coronary artery; evaluation

\section{Introduction}

Kawasaki Disease (KD) as a common type of pediatric disease, the clinical symptoms include of fever and rash, which is a systemic vasculitis. With the continuous improvement of the incidence of Kawasaki disease in recent years, some clinical studies have shown that Kawasaki disease involving the children's cardiovascular system, but also the main reason lead to children with ischemic heart disease. Echocardiography as a common clinical diagnosis of clinical methods, compared with the traditional clinical diagnosis, which has a high diagnostic rate, easy operation and other major features, effectively improve the clinical diagnostic level ${ }^{1}$. Based on this, this study summarizes the relevant literatures on echocardiographic diagnosis of coronary artery changes in children with Kawasaki disease in recent years.

\section{Cardiac changes in the heart of Kawasaki disease}

Kawasaki disease is the pediatric skin and mucosal lymph node syndrome (Mucocutaneous Lymph Node Syndrome,

Copyright (C) 2017 ZHU Lin

doi: http://dx.doi.org/10.18686/aem.v6.83

This is an open-access article distributed under the terms of the Creative Commons Attribution Unported License

(http://creativecommons.org/licenses/by-nc/4.0/), which permits unrestricted use, distribution, and reproduction in any medium, provided the original work is properly cited. 
MCLS), which was reported by a Japanese physician, Kawasaki in 1967. MCLS is a systemic vasculitis for the disease of children with disease, clinical manifestations of acute fever, rash, neck non-agricultural lymph nodes and oral mucosal congestion and so on, more common in more than 3 months, and 5 years of age infants and young children, the proportion of boys is relatively more. Liu Jun and other research scholars in the study of KD, found out by the impact of $\mathrm{KD}$, in the acute phase of small and medium arterial inflammatory response will lead to children with coronary artery intima and the wall layer of varying degrees of damage ${ }^{2}$. After the acute phase, the persistence of the expression of vascular growth factor will increase the intimal hyperplasia and angiogenesis and the coronary wall after healing has also increased the degree of thickening, further reducing the stiffness of the wall and endothelial function.

Some foreign researchers in the KD heart coronary artery changes were analyzed, found that untreated Kawasaki disease cases, about 20 children appeared coronary artery dilatation, but in the course of time 3 weeks to 5 weeks can be self-recovery ${ }^{3}$. Among them, $4 \%$ of children with concurrent coronary artery aneurysm, which is one of the most serious complications of Kawasaki disease, by the impact of coronary artery damage, will lead to myocardial ischemia and even sudden death, a serious threat to the life of healthy children. Coronary artery disease damage caused by KD is mainly manifested in the whole body, small arterial vasculitis, at the same time, according to the different types of coronary artery damage can be divided into four basic stages: (1) The initial stage: 1 week to 2 weeks, mainly for microvascular inflammation, small arterial endositis or perivascular inflammation; (2) Extreme stage: 2 weeks to 4 weeks, mainly for the full-thickness coronary artery infiltration of inflammatory cells, and the formation of the erythematosus, small arterial endometritis or perivascular inflammation; (3) Granulomatosis stage: 4 weeks to 7 weeks, medium-sized artery can be seen in granulation proliferation, and vascular intimal thickening, arteriovenous inflammation, arterial tumor, and some of the thrombus in the tumor will appear thrombosis or vascular stenosis; (4) Mature stage: more than 7 weeks, the performance of the medium-sized artery scar formation, and intimal hypertrophy, stenosis, calcification.

Su Linqiu and Yu Tao stated that cardiovascular damage is common in children with KD type of complications, mainly in the clinical heart disease and coronary artery disease as multiple ${ }^{4}$. Among them, the heart disease occurred in the acute phase of $\mathrm{KD}$, the incidence of up to $90 \%$, this time because of coronary artery disease-like lesions have not yet appeared, so the clinical manifestations of endocardium or myocarditis, usually not causing a greater life threat to children. Coronary artery disease mainly includes coronary arteritis, ischemic lesions and coronary aneurysms. Clinical experience shows that the incidence of coronary artery damage in children with $\mathrm{KD}$ can reach $13 \%$, of which $6 \%$ of children showed permanent damage. In recent years, with the continuous improvement of KD clinical morbidity, how effective diagnosis of coronary artery disease has become the focus of many research scholars and put forward higher requirements for clinical diagnosis.

\section{Evaluation of Cardiac Coronary Artery in Children with Kawasaki}

\section{Disease by Echocardiography}

Clinical studies have shown that KD will have a greater impact on the cardiovascular system of children, especially coronary artery damage (Coronary Artery Damage, CAD), and increasingly aroused the concern of many researchers, but also become the main cause to trigger children of acquired heart disease in current time.

Zhao Lixia and other research scholars in the "The Value of Dynamic Electrocardiogram in Kawasaki Disease Combined with the Diagnosis of Coronary Artery Damage in the Clinical Application" article, selected in January 2012 to August 2014 admitted to 160 cases of children with KD as a research object, all Children were divided into the observation group and the control group, respectively, to take a dynamic electrocardiogram and echocardiography to 
check the coronary artery ${ }^{5}$. The results showed that the number of children with atrioventricular block was $20 \%$ in the observation group underwent echocardiography, which was significantly higher than that in the control group $(2.5 \%)$, and the children with persistent tachycardia accounting for $27.5 \%$, while the control group did not see children with persistent tachycardia, indicating that the diagnosis of dynamic electrocardiogram was significantly better than conventional ECG examination, it is worth to promote in clinical practice. This is also reflected in the study of Guo Q et al., which argues that coronary arteries in KD patients are associated with varying degrees of inflammatory changes, which further lead to coronary artery dilatation or coronary aneurysms, and some patients on this basis, will also be associated with coronary artery stenosis or embolism, the patient's life and health had a huge threat ${ }^{6}$. They also pointed out in the study, KD for the cardiovascular system caused by damage will not only lead to coronary artery inflammation, resulting in patients with myocardial insufficiency, but also will cause other parts of the patient's heart inflammation, such as myocarditis and pericarditis, in severe will also have an impact on the conduction system of the heart. Coronary angiography as a common CAD clinical diagnosis, although it has a high sensitivity, but because of its traumatic examination, combined with KD patients with relatively small age, it can not use coronary angiography for effective examination. In contrast, echocardiography can not only directly reflect the CAD, but also a long time to monitor the patient, play a better clinical diagnostic value.

Yan Gaayong et al. selected 35 children with KD and 2 patients with coronary artery thrombosis as the study object, the general data for children with clinical manifestations and echocardiographic results were analyzed ${ }^{7}$. The results showed that 35 cases of children with echocardiography showed a coronary artery with varying degrees of expansion, 30 cases showed bilateral coronary artery dilatation, 5 cases showed unilateral coronary artery dilatation, 2 cases of coronary artery thrombosis in children with tumor-like expansion, the corresponding wall movement abnormalities. Through the implementation of targeted treatment of children, and its follow-up results after treatment statistics, the results showed that KD children with coronary artery disease showed a dynamic development trend, of which $37.1 \%$ of the coronary artery slightly affects children recovered normal, $42.8 \%$ of children with the expansion of the phenomenon has been effectively alleviated, $14.3 \%$ showed coronary aneurysm-like expansion, and no significant changes before treatment. It can be found that echocardiography can effectively diagnose the changes of coronary artery in children with Kawasaki disease, and have a positive effect on reducing the incidence of complications in children and has a high clinical value.

Xie Yi and others also found that KD disease will have serious damage to children with coronary artery, but also the main cause of coronary artery disease in children ${ }^{8}$. All of the children were diagnosed according to the diagnostic criteria for coronary artery dilatation, coronary aneurysm, and coronary thrombosis issued by the Japanese Ministry of Health in 1984 during the clinical diagnosis of 42 children with KD. According to the survey, 42 cases of children with coronary artery dilatation (59.52\%), 4 cases of tumor-like expansion, 3 cases of stenosis and thrombosis in 1 case. According to the clinical test results of children, the implementation of the corresponding drug treatment, after 3 months after the review, the results show that 25 cases of coronary artery dilatation in all children with coronary artery retraction, wall thinning, and visible Intimal clear and smooth. Among the 4 patients with coronary aneurysms, one patient was with thrombosis and converted to an old thrombus. After 1 year, the thrombus disappeared. The remaining 3 cases, 1 case in 3 years after the review still showed mild expansion, the remaining 2 cases returned to normal. Thus, echocardiography can be found in children with KD coronary artery abnormalities, for the clinical diagnosis and treatment of the effective development of a positive impact.

\section{Conclusion}

In a nutshell, echocardiography is now widely used as a modern diagnostic method, which can effectively achieve the diagnosis of children with Kawasaki disease, early detection of complications, through early treatment and prognosis, 
healthy recovery has a positive impact. At the same time, through the treatment of children with Kawasaki disease after echocardiography diagnosis, will further understand the recovery of coronary artery to ensure that the clinical treatment of targeted and effectively improve the level of clinical treatment, which should be widely used and promoted.

\section{References}

1. Wang Renjian, Huang Min, Xie Lijian, et al. Application of Two-Dimensional Speckle Tracking Echocardiography in the Assessment Of Cardiomyopathy in Children with Kawasaki Disease [J]. Journal of International Pediatrics, 2016,43 (8): 638-642.

2. Liu Jun, Yu Minghua, Xu Kaiyuan, et al. 320 Square Volumetric CF Coronary Angiography in Coronary Artery Aneurysm of Kawasaki Disease in Long-Term Follow-Up [J]. Journal of Clinical Pediatrics, 2015,33 (7): 601 -604.

3. Qin F, Huang X, Haibin M O. Echocardiography, Coronary Angiography and Diagnosis of Coronary Heart Disease Comparative Analysis [J]. Journal of Medical Theory \& Practice, 2015, 11 (4): 157-158.

4. Su Linqiu, Yu Tao. Echocardiography in Children with Kawasaki Disease Coronary Artery Disease Diagnosis and Prognosis of the Value of the Application [J]. Medicine frontier, 2016,6 (13): 203-204.

5. Zhao Lixia, Zhao Liping, Shen Lin. Dynamic Electrocardiogram in the Diagnosis of Kawasaki Disease Combined with Coronary Artery Disease in the Clinical Application Value [J]. Chinese Journal of Maternal and Child Health, 2016,27 (1): 64-66.

6. Guo Q, Zhang Y, Electrocardiogram T. Supersonic and Enchanted the Graph on the Analysis of the Diagnosis of Right Ventricular Cardiomyopathy Arrhythmia [J]. China Health Standard Management, 2015, 26 (8): 252-256.

7. Yan Jia-yong, Liu Yang, Xu Jing. Children with Kawasaki Disease Coronary Artery Disease and Its Complications of Ultrasound Diagnosis and Value [J]. Integrative Medicine cardiovascular disease electronic magazine, 2015,3 (30): 140-141.

8. Xie Yi, Huang Meifeng, Peng Xuefang, et al. Diagnosis of Coronary Artery Lesion in Children with Kawasaki Disease by Echocardiography [J]. Journal of Youjiang Medical College for Nationalities, 2016,38 (2): 193-194. 